\title{
Palisaded encapsulated neuroma of the upper lip
}

\author{
Kayo Kuyama ${ }^{1,2 *}$, Hirotaka Oomine ${ }^{3}$, Yan Sun ${ }^{1}$, Masanobu Wakami ${ }^{4}$, Hirotsugu Yamamoto ${ }^{1,2}$ \\ ${ }^{1}$ Department of Oral Pathology, Nihon University School of Dentistry at Matsudo, Matsudo, Japan \\ ${ }^{2}$ Department of Diagnostic Pathology, Hospital of Nihon University School of Dentistry at Matsudo, Matsudo, Japan \\ ${ }^{3}$ Department of Maxillo-Facial Orthodontics, Nihon University School of Dentistry at Matsudo \\ ${ }^{4}$ Department of Crown and Bridge Prosthodontics, Nihon University School of Dentistry at Matsudo, Matsudo, Japan \\ Email: "kuyama.kayo@nihon-u.ac.jp
}

Received 12 July 2012; revised 16 August 2012; accepted 26 August 2012

\begin{abstract}
Palisaded encapsulated neuroma (PEN; solitary circumscribed neuroma) is a benign and hyperplastic lesion consisting of Schwann cells. PEN of the lower lip was reported by Tomich and Moll [1] 35 years ago. However, the accumulation of the information about PEN which occurred in the oral mucosa was not enough. This article describes a case of a PEN on the upper lip of a 41-year-old woman. The lesion with 0.7 cm diameter was performed excisional biopsy. Histologically, the tumor was almost circumscribed by thin fibrous capsule, and consisted of diffusely and dense proliferation of the spindle shape cells arranged in interlacing fascicles. Focal suggestions of nuclear palisaded growth were indicated within the tumor. Immunohistochemically, the fascicles of tumor cells were positive for S-100 protein, and vimentin and negative for $\alpha$-actin and GFAP. A few numbers of axons were demonstrated by anti-neurofilament antibody in this lesion. Therefore, the definitive diagnosis was PEN.
\end{abstract}

Keywords: Palisaded Encapsulated Neuroma; Solitary Circumscribed Neuroma; Immunohistochemistry

\section{INTRODUCTION}

The palisaded encapsulated neuroma (PEN) is an uncommon entity, first described as a series of 44 cases of the skin in 1972 by Reed et al. [2]. PEN is a hyperplastic lesion, nodular appearance, consisting of Schwann cells with completely/incompletely encapsulated. The Schwann cells, often aligned and fasciculated focally showed nuclear palisading. The first occurs to the face and the 2nd frequency is the oral mucosa. In 1976, PEN of the lower lip was reported by Tomich and Moll [1]. However, accumulation of the information about PEN which occurred on the oral mucosa is not enough.

*Corresponding author.
This article describes a case of a PEN on the upper lip of a 41-year-old woman. Clinical, histopathological and immunohistochemical findings were shown, and review of literature also performed concerning about this characteristic lesion including differential diagnosis.

\section{CASE REPORT}

A healthy 41-year-old woman presented with a complaint of a gradual painless swelling measuring $0.7 \mathrm{~cm}$ in diameter of 2 years duration on the inside of the upper lip. The lesion was painless, firm and the mucosa overlying the lesion appeared normal without ulcerations or mucosal discoloration. Her medical and dental histories were uneventful. The patient underwent excision of the upper lip and excisional biopsy was performed. She tolerated the procedure well and had no recurrences 5 years after the operation. The solitary small tumor was clinically thought to be a fibroma.

The specimen performed histopathological diagnosis with hematoxylin and eosin staining (H.E.) by 2 oral pathologists. Immunohistochemical studies were conducted using the EnVision + Polymer System (Dako Glostrup, Denmark), and primary antibodies used were directed against the following antigens: S-100 (1:500; Dako Glostrup, Denmark); Actin ( $\alpha$-SMA, 1A4, 1:100; Dako Glostrup, Denmark); Neurofilament protein (NF, 2F1, 1:100; Dako Glostrup, Denmark); Neuron-specific enolase (NSE，BBS/NC/VI-H14，1:100; Dako Glostrup, Denmark); Glial fibrillary acidic protein (GFAP, 6F2, 1:100; Dako Glostrup, Denmark)and Vimentin (Vim 3B4, 1:100; Dako Glostrup, Denmark). The sections were developed in a solution of 3.3'-dianibobenzidine tetrahydrochloride (DAB). Finally, all sections were counterstained with Mayer's hematoxylin. Positive controls for S-100, NF, NSE, GFAP and vimentin were specimens of normal peripheral nerve system, that for $\alpha$-SMA was specimens of leiomyoma. For evaluation of the immunohistochemical staining technique, as a negative control, mouse and rabbit universal negative controls (Dako Glostrup, Denmark) were used during the staining pro- 
cedure instead of primary antibodies.

\subsection{Histopathological Findings}

Biopsy of the specimen revealed a whitish-gray cutsurface. Histologically, the tumor was almost circumscribed by thin fibrous capsule (Figures 1(a) and (b)), and consisted of diffusely and dense proliferation of the spindle or elongated shape cells with scarce cytoplasm arranged in interlacing fascicles and indistinct cell borders (Figures 1(c) and (d)). Small neuroid structures were scattered and focal suggestions of nuclear palisaded growth was indicated within the tumor (Figure 1(c)). Individual spindle shape cells exhibited elongated, sinuous to ovoid nuclei with occasional nuclear holes ("Lochkerne (nuclear holes)”) (Figure 1(d)). There was no atypia or mitotic evidence of malignancy. The stroma of the lesion was minimal and eosinophilic/PAS positive in appearance contained few capillaries and scattered mast cells (Figures 1(e) and (f)). The histopathologic diagnosis was consistent with a palisaded encapsulated neuroma.

\subsection{Immunohistochemical Findings}

Immunohistochemicallly, the fascicles of tumor cells were positive for S-100 protein (Figure 1(g)), vimentin (Figure 1(h)) and negative for $\alpha$-actin and GFAP (Figure 1(i)). A few numbers of small neuroid structure and axons were demonstrated by NSE (Figure 1(j)) and NF (Figure 1(k)) in this lesion, respectively. These results indicate that the tumor is composed of cells of schwannian differentiation and they also indicate the presence of axons. Therefore, the definitive diagnosis was PEN. There was no relationship with von Recklinghausen's disease, and none of the lesions recurred after excision.

\section{DISCUSSION}

PEN is a benign, morphologically characteristic skin or mucosal neuroma. The majority of PEN was occured in the oral mucosa with the most frequent [3]. In the mucosal lesion, other literatures expressed that PEN had occurred on the mucocutaneous junctions, for example, eyelid, nasal fossa, and oral mucosa overwhelmingly in the face, on the contrary [4-6]. Accumulation of information was scarce, though several series have detailed its clinicopathologic and immunohistochemical features of PEN on the oral mucosa. To further accumulate of a report of this characteristic lesion, we herein describe a case of PEA with clinical, histopathologic, and immunohistochemical features of this entity.

The results of the clinico-pathological findings by review of literature were shown in Table 1. The incidence rate of $\mathrm{PEN}$ in the oral mucosal biopsy specimens were $0.04 \%$ to $0.05 \%$ in the previous reports [4,7], and PEN accounts for approximately $22 \%$ to $25 \%$ of all peripheral nerve origin in the oral cavity [4,7]. The mean age of PEN on the oral mucosa was between 37 to 54 years $[3,4,7,8]$ and it was almost the same tendency that of the skin's as between 40 and 60 years [2]. As for sex difference, men were almost predominant to women $[3,7,8]$.

Clinically, all tumors were nodular in appearance [3] with small and most often painless [3]. Multifocal lesions were rare. The tumor were solitary, sessile, firm, normal mucosal colored, between 2 to $6 \mathrm{~mm}$ of diameter, and the size of which never exceeded $1.0 \mathrm{~cm}$ in greatest dimension [3]. The most common clinical diagnosis was "focal fibrous hyperplasia (fibroma)" [7]. None of the patients with PEN had neurofibromatosis or multiple endocrine neoplasia syndrome type III [7]. The clinical findings of this case were concordance with the previous reports except for female.

Histologically, PENs were partially or completely encapsulated and composed of fiscicles of spindle-shaped cells that might show some nuclear palisading. Although mild nuclear pleomorphism, might be seen [9], significant atypia and mitotic activity were generally lacking. Fletcher noted that most lesions were not fully encapsulated. Despite their original name, many cases were incompletely encapsulated and showed only focally nuclear palisading [10]. The name "solitary circumscribed neuroma” also existed from such a histological background.

Oral benign peripheral nerve tumor/tumor-like lesions were listed in Table 2 [11]. Based on clinical and histologicl similarities, the differential diagnosis of PEN on the oral mucosa includes neurofibroma, schwannoma, traumatic neuroma and leiomyoma [12]. Neurofibromas are unencapsulated and consisting of mixture of Schwann cells, perineurial cells, and endoneurial fibroblasts [11]. The lesion looks more loosely textured with myxoid stroma than PEN and shows a delicate fibrillary pattern of collagen deposition. Schwannomas are usually submucotaneous, contain Antoni A and B types with Verocay bodies and lack axons [13]. Histologically, PENs have distinctive features, consisting of a proliferation of Schwann cells and a certain amount of axons, becoming reliable distinction from schwannomas and neurofibromas. In addition, since most of the cells in PENs are normal Schwann cells, nuclear sizes are larger and more homogeneous than those of neurofibromas. Immunohistochemical analyses demonstrate strong positive tumor cell of PEN staining for S-100 protein, and vimentin characteristic of Schwann cell differentiation [12]. Marked S-100 immunoreactivity of schwannomas and PENs were exhibited in contrast to neurofibroma Koutlas IG. Furthermore, it was negative of PEN for GFAP and $\alpha$-SMA. The immunohistochemical results of the present case were supported definitive diagnosis of PEN. Moreover, the positive axon staining of PENs may be useful in differentiating PENs from schwannomas. 

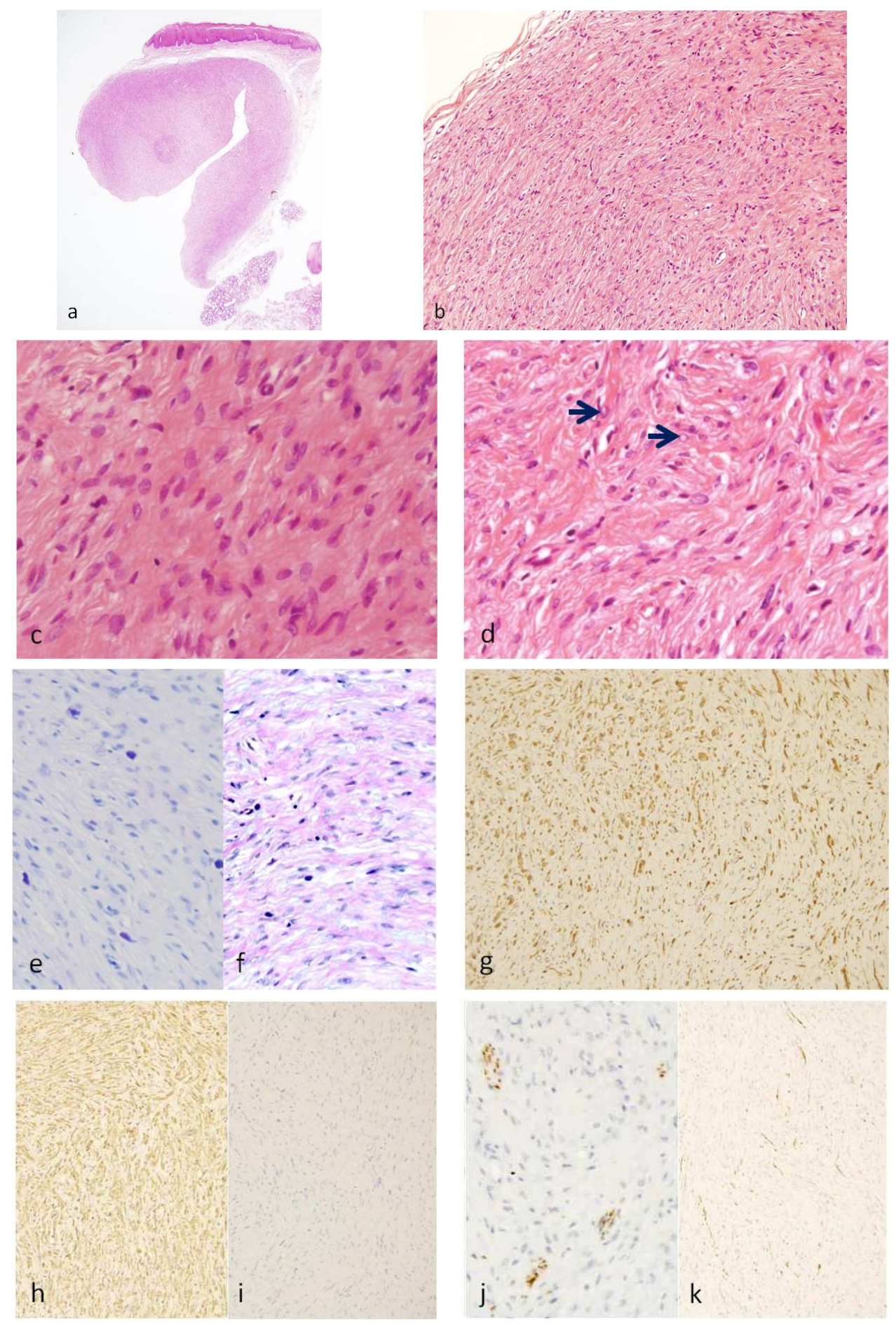

Figure 1. Histopathological and immunohistochemical figures: (a) A irregular form of tumor-like lesion was occupied the majority of the biopsy (H.E. $\times 2$ ); (b) The lesion was well circumscribed and partly surrounded by thin capsule; (c) Nuclear palisading and "Verocay-like bodies" were focally presented in the lesion (H.E. ×40); (d) Cellular proliferations of spindle shape cells was formed microfascicles and nuclei with occasional nuclear holes (arrow, "Lochkerne (nuclear holes)") were shown (H.E. $\times 60$ ); (e) A small number of mast cells diffusely appeared in the lesion $(\mathrm{TB}, \mathrm{pH} 4.1 \times 40)$; (f) Storoma was composed this fibrous tissue (PAS $\times 40$ ); (g) Strong positive immunoreactions for S-100 (×40); (h) Positive reaction for vimentin $(\times 20)$; (i) Negative immunoreactions for GFAP $(\times 40)$; $(\mathrm{j})$ Small neuroid structures were observed inside the lesion $(\times 20)$; $(\mathrm{k})$ Positive NF staining for axons were scattered $(\times 20)$. 
Table 1. Review of literature.

\begin{tabular}{|c|c|c|c|c|}
\hline Large Item & No & Clinical Item & Value & Reference \\
\hline \multirow[t]{5}{*}{ Gender ratio } & $12^{*}$ & Male: Female ratio (\%) & $40 」 \exists 0$ & [4] \\
\hline & $13^{*}$ & Male: Female ratio (\%) & $77 」 \exists 3$ & [7] \\
\hline & $16^{*}$ & Male: Female ratio (\%) & $56 」 \exists 38^{\mathrm{a}}$ & [8] \\
\hline & $54^{*}$ & Male: Female ratio (\%) & $70 」 \exists 30$ & {$[3]$} \\
\hline & $1^{*}$ & Female & --- & Kuyama et al. \\
\hline \multirow[t]{5}{*}{ Age } & $12^{*}$ & Average age & 54 yrs & {$[4]$} \\
\hline & $13^{*}$ & Average age & 51 yrs & [7] \\
\hline & $16^{*}$ & Average age & $37 \mathrm{yrs}$ & {$[8]$} \\
\hline & $54^{*}$ & Average age & 48 yrs & [3] \\
\hline & $1^{*}$ & 。 & $41 \mathrm{yrs}$ & Kuyama et al. \\
\hline \multirow[t]{20}{*}{ The site of the lesion } & $12^{*}$ & Palate & $41.7 \%$ & [4] \\
\hline & 。 & Upper lip & $33.3 \%$ & \\
\hline & ○。 & Tongue & $16.7 \%$ & \\
\hline & $\therefore$ & NS & $8.3 \%$ & $\therefore$ \\
\hline & $13^{*}$ & Palate & $77.0 \%$ & [7] \\
\hline & 。 & Upper lip & $7.7 \%$ & \\
\hline & $\circ$ & Lower lip & $7.7 \%$ & \\
\hline & $\circ$ & Gingiva & $7.7 \%$ & $\circ$ \\
\hline & $16^{*}$ & Palate & $68.8 \%$ & {$[8]$} \\
\hline & 。 & Tongue & $12.5 \%$ & \\
\hline & $\circ$ & Buccal mucosa & $6.3 \%$ & \\
\hline & $\therefore$ & Lip & $6.3 \%$ & \\
\hline & $\ldots$ & Gingiva & $6.3 \%$ & 。 \\
\hline & $54^{*}$ & Palate & $57.4 \%$ & [3] \\
\hline & $\therefore$ & Gingiva & $20.4 \%$ & \\
\hline & 。 & Tongue & $7.4 \%$ & \\
\hline & 。 & Upper lip & $5.6 \%$ & \\
\hline & $\therefore$ & Lower lip & $9.3 \%$ & \\
\hline & 。 & Buccal mucosa & $1.9 \%$ & ○ \\
\hline & $1^{*}$ & Upper lip & $\therefore$ & Kuyama et al. \\
\hline \multirow[t]{5}{*}{ Duration } & $12^{*}$ & $0-5 \mathrm{yrs}$ & $41.7 \%$ & {$[4]$} \\
\hline & 。 & $6-10$ yrs & $16.7 \%$ & \\
\hline & 。 & $>11 \mathrm{yrs}$ & $16.7 \%$ & \\
\hline & $\ldots$ & NS & $25.0 \%$ & $\ldots$ \\
\hline & $1^{*}$ & 2 yrs & ○ & Kuyama et al. \\
\hline \multirow[t]{4}{*}{ Clinical diagnosis } & $12^{*}$ & Papilloma & $16.70 \%$ & [4] \\
\hline & 。 & Fibroma & $83.30 \%$ & ○ \\
\hline & $13^{*}$ & Fibroma & almost $^{\mathrm{b}}$ & [7] \\
\hline & $1^{*}$ & Benign tumor & --- & Kuyama et al. \\
\hline
\end{tabular}

${ }^{*}$ Case number; ${ }^{\mathrm{a} G e n d e r ~ u n k n o w n ~ i n ~ o n e ~ c a s e ; ~}{ }^{\mathrm{b}}$ Case number was unknown. 
Table 2. Contents of benign tumor/tumor-like lesions of the peripheral nerve.

\begin{tabular}{l}
\hline 1. Reactive lesions \\
\hline 1) Traumatic neuroma \\
2) Nerve cyst \\
2. Inflammatory and infectious lesions simulating tumors of the nerve \\
1) Inflammatory pseudotumor of nerve \\
2) Sarcoidosis of peripheral nerve \\
3. Hyperplastic lesions \\
1) Palisaded encapsulated neuroma (PEN) \\
2) Mucosal neuromatosis (MEN) \\
3) Localized hypertrophic neuropathy \\
4. Hamartoma and Choristoma \\
5. Tumor \\
1) Schwannoma \\
2) Neurofibroma \\
3) Miscellaneous benign neurogenic tumors
\end{tabular}

Traumatic neuroma is a non-neoplastic, disorganized proliferation of axons, Schwann cells, and perineurial cells, all in a fibrocollagenous stroma [11]. Although traumatic neuromas display abundant Schwann cells and axons, inflammatory cells and scaring are also visualized. Leiomyomas are distinguished by the presence of muscle cells in the tumor and positive staining for $\alpha$-SMA.

The proposed pathophysiology of the PEN was suggested as a hamartomatous growth of Schwann cells predominating over the number of axons, however, the stimulus that triggers these Schwann cells to proliferate has not been identified [14]. On the contrary, recurrent trauma plays an etiologic role of the hyperplastic lesion of PEN [9,12,15]. Such dilated capillaries and venuses have been reported to occur in PENs with recurrent trauma. However, such a remarkable blood vessel reaction was not accepted with this case. The further examination is required for a cause of a disease.

\section{REFERENCES}

[1] Tomich, C.D. and Moll, M.C. (1976) Palisaded, encapsulated neuroma of the lip. Journal of Oral Surgery, 34, 265-268.

[2] Reed, R.J., Fine, R.M. and Meltze, H.D. (1972) Palisaded, encapsulated neuromas of the skin. Archives of Dermatology, 106, 865-870.

doi:10.1001/archderm.1972.01620150051016

[3] Koutlas, I.G. and Scheithauer, B.W. (2010) Palisaded encapsulated ("solitary circumscribe") neuroma of the oral cavity: A review of 55 cases. Head and Neck Pathology, 4, 15-26. doi:10.1007/s12105-010-0162-x

[4] Magnusson, B. (1996) Palisaded encapsulated neuroma (solitary circumscribed neuroma) of the oral mucosa. Oral Surgery, Oral Medicine, Oral Pathology, Oral Radiology and Endodontology, 82, 302-304.

[5] Mayorga, M., Acebo, E. and Val-Bernal, J. (1998) Pali- saded encapsulated neuroma of the nasal fossa. Otolaryngology—Head and Neck Surgery, 119, 141-143. doi:10.1016/S0194-5998(98)70196-2

[6] Navarro, M., Vilata, J., Requena, C. and Aliaga, A. (2000) Palisaded encapsulated neuroma (solitary circumscribed neuroma) of the glans penis. British Journal of Dermatology, 142, 1061-1062. doi:10.1046/j.1365-2133.2000.03507.x

[7] Chauvin, P.J., Wysocki, G.P., Daley, T.D. and Pringle, G.A. (1992) Palisaded encapsulated neuroma of oral mucosa. Oral Surgery, Oral Medicine, Oral Pathology, 73, 71-74. doi:10.1016/0030-4220(92)90158-M

[8] Chrysomali, E., Papanicolaou, S.I., Dekker, N.P. and Regezi, J.A. (1997) Benign neural tumors of the oral cavity: A comparative immunohistochemical study. Oral Surgery, Oral Medicine, Oral Pathology, Oral Radiology and Endodontology, 84, 381-390. doi:10.1016/S1079-2104(97)90036-6

[9] Dover, J.S., From, L. and Lewis, A. (1989) Palisaded encapsulated neuromas: A clinicopathologic study. Archives of Dermatology, 125, 386-389.

[10] Fletcher, C.D.M. (1989) Solitary circumscribed neuroma of the skin (so-called palisaded, encapsulated neuroma): A clinicopathologic and immunohistochemical study. American Journal of Surgical Pathology, 13, 574-580. doi:10.1097/00000478-198907000-00005

[11] Scheithauer, B.W., Woodruff, J.M. and Erlandson, R.A. (1997) Reactive lesions, inflammatory and infectious lesions simulating tumors of the nerve, hyperplastic lesions, hamartoma and choristoma, schwannoma, neurofibroma and miscellaneous benign neurogenic tumors. In: Tumors of the Peripheral Nerve System, Armed Forces Institute of Pathology, Washington DC, 25-282.

[12] Argenyi, Z. (1990) Immunohistochemical characterization of palisaded, encapsulated neuroma. Journal of $\mathrm{Cu}$ taneous Pathology, 17, 329-335. doi:10.1111/j.1600-0560.1990.tb00108.x

[13] Zelgar, B., Stainer, H., Kutner, H., Rütten, A. and Zelger, B. (1997) Verocay body-prominent cutaneous schwan- 
noma. American Journal of Dermatopathology, 19, 242249. doi:10.1097/00000372-199706000-00007

[14] Dover, J., From, L. and Lewis, A. (1989) Palisaded encapsulated neuromas: A clinicopathologic study. Archives of Dermatology, 125, 386-389. doi:10.1001/archderm.1989.01670150076012

[15] Albrecht, S., Kahn, H.J. and From, L. (1989) Palisaded encapsulated neuroma: An immunohistochemical study. Modern Pathology, 2, 403-406. 